\title{
Safety and Efficacy of Aminophylline in Intrathecal Methotrexate-related Neurological Toxicity in Large Pediatric Oncology Centre
}

\author{
Wajeeha Razi ${ }^{1}$, Anwar UI Haque ${ }^{2}$, Hassaan Sadiq ${ }^{1}$, Raza Ullah ${ }^{3}$, Naeem Jabbar ${ }^{4}$ and Sadiq Mirza ${ }^{2}$ \\ ${ }^{1}$ Department of Paediatrics, The Indus Hospital, Karachi, Pakistan \\ ${ }^{2}$ Department of Paediatrics Critical Care Medicine, The Indus Hospital, Karachi, Pakistan \\ ${ }^{3}$ Department of Pharmacy, The Indus Hospital, Karachi, Pakistan \\ ${ }^{4}$ Department of Paediatric Hematology Oncology, The Indus Hospital, Karachi, Pakistan
}

\begin{abstract}
Children with acute lymphoblastic leukemia, receiving intrathecal methotrexate (IT-MTX), develop acute and subacute neurological dysfunction in 3-15\% of cases. Altered level of consciousness, seizure, and stroke-like manifestations are among the most common presentations. MRI of the brain in an early stage is consistent with white matter leukoencephalopathy. There are no specific guidelines for the treatment of such a syndrome. An elevated cerebrospinal fluid (csf) adenosine concentration causes vasodilatation in the brain and leads to cerebral ischemia, which may be reduced by aminophylline infusion. The study is a retrospective data analysis in which electronic data records of 30 patients, collected from September 2017 - August 2019. The primary objective of the study was to evaluate the safety and efficacy of aminophylline in IT-MTX induced neurotoxicity. Aminophylline infusion was used in 30 patients with IT-MTX neurotoxicity. Twenty-five patients (83.33\%) showed dramatic improvement of neurologic signs and symptoms within 48 hours. Aminophylline was, hence, coined as a salvageable therapy. No noticeable side effects were observed during treatment with aminophylline infusion.
\end{abstract}

Key Words: Intrathecal methotrexate, Neurotoxicity, Children, Cancer, Aminophylline.

How to cite this article: Razi W, Haque AU, Sadiq H, Ullah R, Jabbar N, Mirza S. Safety and Efficacy of Aminophylline in Intrathecal Methotrexate-related Neurological Toxicity in Large Pediatric Oncology Centre. J Coll Physicians Surg Pak 2021; 31(04):481-484.

Acute lymphoblastic leukemia (ALL) is the most common hematological malignancy in pediatrics, and accounts for more than $50 \%$ of childhood cancer in Pakistan. ${ }^{1}$ Methotrexate (MTX) is an anti-folate agent and chemotherapeutic agent in many established protocols to treat ALL as well as other malignancies. It has many adverse effects, the most important being neurotoxicity and nephrotoxicity. The types of MTX-neurotoxicity are acute, subacute, and chronic toxicity, based on temporal presentation. Acute and subacute neurotoxicity due to MTX can present within a few days to weeks after high-dose intravenous MTX or intrathecal MTX. Spectrum of clinical manifestation varied from mild symptoms to life-threatening events, such as headache, vomiting, seizure, altered mental status (assessed by Glasgow Coma Scale), blurring of vision to acute blindness and focal neurological deficit.

Correspondence to: Dr. Sadiq Mirza, Department of Critical Care Medicine, The Indus Hospital, Karachi, Pakistan E-mail: sadiq.mirza@tih.org.pk

Received: June 02, 2020; Revised: December 01, 2020;

Accepted: December 05, 2020

DOI: https://doi.org/10.29271/jcpsp.2021.04.481
MRI of the brain with diffusion-weighted imaging (DWI) is the imaging modality of choice for diagnosis of IT-MTX neurotoxicity, which described as leukoencephalopathy. ${ }^{2}$ However, there is no standard guideline for the treatment of this devastating complication as a result of the administration of IT-MTX.

Few published reports demonstrated that the intravenous aminophylline ameliorates the neurological signs and symptoms related to IT-MTX ${ }^{3,4}$ The authors retrospectively reviewed the onco-critical care experience of this specific complication, including initial neurological signs and symptoms, clinical course, imaging, and effects of aminophylline in the resolution of neurological dysfunction.

The objective of this study was to evaluate the efficacy and safety of intravenous aminophylline as a salvageable therapy in pediatric oncological cases suffering from intrathecal MTX-related neurological toxicity.

Electronic medical records of all children (age: 2-16 year) with leukemia and lymphoma were retrospectively reviewed, who developed acute and subacute neurotoxicity after IT-MTX and admittedintoaclosed, multidisciplinarymedical-surgical pediatric intensive care unit (PICU) of the Indus Hospital Karachi with a large pediatric oncology centre, from September 2017 to August 2019. The dose of IT-MTX given was calculated by the 
oncology team as per standard guidelines for pediatric malignancies. After excluding other causes of neurological dysfunction, like central nervous system (CNS) infections, cranial irradiation, or CNS disease through other investigations (CSF analysis, neuroimaging), aminophylline therapy was targeted to treat neurotoxicity signs and symptoms caused by IT-MTX (loss of consciousness, seizures, hypertension, Table I). All patients received supportive care and neuroprotective strategies with intense monitoring in PICU. These neuroprotective strategies, which include keeping the head supine and raised by 30 degrees, regulating sodium levels and blood pressure, preventing and controlling fever and seizures, are part of the standardised protocol of all patients with neurological insult. Patients who showed IT-MTX-induced clinical neurotoxicity, despite these measures were included in the study. Each patient received intravenous aminophylline $(2.5 \mathrm{mg} / \mathrm{kg})$ as a loading dose over 20-30 minutes, followed by an infusion of intravenous aminophylline (at $0.5 \mathrm{mg} / \mathrm{kg} /$ hour) for 24 hours. ${ }^{3}$ There were no side effects (headache, nausea, vomiting, abnormal heart rhythm, or seizures) observed after intravenous aminophylline. The primary outcome is defined as the reversibility of neurological dysfunction back to normal baseline neurological status within 48 hours. All data were entered into a structured data collection sheet, including demographic, clinical, and outcome variables, and transferred into SPSS version 22.0 for descriptive analysis. Qualitative variables like gender, diagnostic category, and efficacy were expressed as frequency and percentage as appropriate while the quantitative variables were expressed as mean with standard deviation (mean \pm SD) like age. This study was approved by the Institutional Ethical Committee (Study IRB No. IRD_IRB_2020_ 04_006).

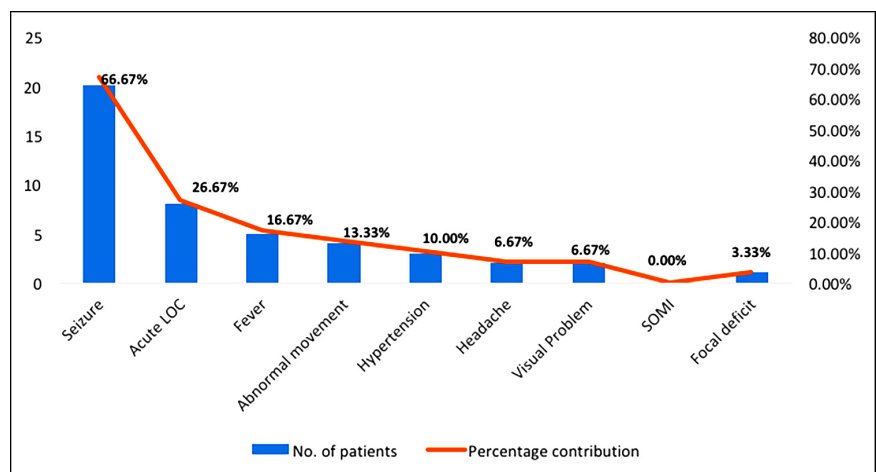

Figure 1: Percentages of different clinical presentation of methotrexate neurotoxicity.

Thirty patients were enrolled. The mean age was 8.37 ( \pm 3.91 ) years and males were $70 \%(n=21)$. Patients' characteristics were described in Table I. Most of the cases were acute leukemia and lymphoma. Most patients ( $n=20,66.67 \%$ ) had seizures, followed by altered mental status $(n=8$, $26.67 \%$ ). Figure 1 displays the frequency of neurological signs and symptoms due to IT-MTX neurotoxicity. Cerebrospinal fluid analysis and head CT-scan were done on 20 and 13 patients respectively, which were normal study. Out of $17 \mathrm{MRI}$ of the brain, 12 patients had findings consistent with IT-MTX toxicity. ${ }^{2}$
It is due to the non-availability of MRI in the facility and most of them were done late and missed classical features. Twenty-five patients (83.33\%) completely recovered with aminophylline therapy, while the remaining five patients $(n=5,16.67 \%)$ did not recover and expired. All 25 patients ( $n=25,83.33 \%$ ) recovered within 48 hours after the administration of intravenous aminophylline infusion. There were no side effects, like headache, nausea, vomiting, abnormal heart rhythm, or seizures, observed after intravenous aminophylline.

Table I: Patients' characteristics having methotrexate neurotoxicity $(n=30)$.

\begin{tabular}{|c|c|c|}
\hline Variables & $\begin{array}{l}\text { Values (in } \\
\text { numbers) }\end{array}$ & Values (in \%) \\
\hline Age (years) & $8.37 \pm 3.91$ & \\
\hline \multicolumn{3}{|l|}{ Gender } \\
\hline Male & 21 & $70.00 \%$ \\
\hline Female & 9 & $30.00 \%$ \\
\hline \multicolumn{3}{|l|}{ Diagnosis } \\
\hline Leukemia & 23 & $76.67 \%$ \\
\hline Lymphoma & 07 & $23.33 \%$ \\
\hline \multicolumn{3}{|l|}{ Phases } \\
\hline Induction & 8 & $26.67 \%$ \\
\hline Post-induction & 22 & $73.33 \%$ \\
\hline \multicolumn{3}{|l|}{ Clinical variables } \\
\hline Seizure & 20 & $66.67 \%$ \\
\hline $\begin{array}{l}\text { Acute loss of consciousness } \\
(\text { LOC) }\end{array}$ & 8 & $26.67 \%$ \\
\hline Fever & 5 & $16.67 \%$ \\
\hline Abnormal movement & 4 & $13.33 \%$ \\
\hline Hypertension & 3 & $10.00 \%$ \\
\hline Headache & 2 & $6.67 \%$ \\
\hline Visual Problem & 2 & $6.67 \%$ \\
\hline $\begin{array}{l}\text { Signs of meningeal irritation } \\
\text { (SOMI) }\end{array}$ & 0 & $0.00 \%$ \\
\hline Focal deficit & 1 & $3.33 \%$ \\
\hline CSF analysis & 20 (all negative) & $66.67 \%$ \\
\hline CT head & 13 (normal) & $43.33 \%$ \\
\hline MRI head & 17 & $56.67 \%$ \\
\hline \multicolumn{3}{|c|}{ Time } \\
\hline \multicolumn{3}{|c|}{ Duration of symptom after IT MTX } \\
\hline Within 48-hours & 16 & $53.33 \%$ \\
\hline After 48- hours & 14 & $46.67 \%$ \\
\hline \multicolumn{3}{|l|}{ Duration took for recovery } \\
\hline Within 48-hours & 25 & $83.33 \%$ \\
\hline After 48-hours & 00 & $0.00 \%$ \\
\hline \multicolumn{3}{|l|}{ Outcome } \\
\hline Full recovery & 25 & $83.33 \%$ \\
\hline Expiries (deaths) & 05 & $16.67 \%$ \\
\hline
\end{tabular}

Acute neurological disorders in children undergoing chemotherapy for malignancy include infections, neoplastic, hemostatic (bleeding/thrombosis), metabolic, and drug toxicities. Methotrexate-induced toxicity has been extensively described in the literature. The acute leukoencephalopathy related to IT-MTX is reported in $3-15 \%$ in published clinical reports. ${ }^{4}$ Twenty-five $(83.33 \%)$ patients completely recovered from IT-MTX-induced neurotoxicity after administration of aminophylline. Bernini JC et al first described the experience of using intravenous aminophylline as a treatment of this complication in 1995, where four out of six patients recovered after aminophylline injection. All six patients (age 3-16 years) were known cases of acute lymphoblastic leukemia, who developed 
neurotoxicity after IT-MTX and had not recovered from standard therapy. ${ }^{3}$ In another case-series, children who developed this leukoencephalopathy after IT-MTX developed acute or subacute neurologic disorder like headache, altered mental status, seizure and stroke-like manifestation. White matter parenchymal damaged most of them, improved after receiving aminophylline infusion. ${ }^{4}$ Ganesan $\mathrm{P}$ et al. reported a 24 -year patient who developed quadriparesis and cranial nerve palsies recovered after aminophylline administration. He was initially given dexamethasone and leucovorin to treat neurotoxicity and the condition got worse, but the symptoms improved after aminophylline infusion. ${ }^{5}$ All these clinical reports suggest that intravenous aminophylline causes complete and rapid amelioration of neurological symptoms related to IT-MTX neurotoxicity. ${ }^{3-5}$

In the present cohort, most of the patients presented with generalised dysfunctions like seizures and altered mental status after the administration of IT-MTX. Similar clinical symptoms were seen in a case series; six patients (age 3-16 years), who presented with IT-MTX neurotoxicity demonstrated symptoms including headache, nausea, vomiting, and lethargy. However, there were variable presentations of IT-MTX neurotoxicity from generalised dysfunction to focal neurological deficit in various case reports. ${ }^{4,5}$

MRI of the brain is the imaging modality of choice to diagnose methotrexate-induced neurotoxicity. The most common findings in MRI-DWI have restricted diffusion with low apparent diffusion coefficient (ADC) in the periventricular area of the deep white matter of cerebral hemispheres either unilaterally or bilaterally, particularly in the centrum semiovale. Sometimes the lesion may extend to involve frontal or occipital subcortical white matter.

These findings are usually associated with cytotoxic edema, which is transient and seen only in the early stages of acute methotrexateneurotoxicity and described astransient leukoencephalopathy. ${ }^{2}$ After an MRI facility was made available at the hospital, patients underwent MRI scans after the administration of aminophylline. The abnormality in scans after IT-MTX administration was that of white matter changes and some of these patients who had recovered from neurotoxicity clinically also showed normal scans within 48 hours of infusion. Thirteen out of 25 patients, who showed clinical recovery, also had a normal CT scan 48 hours after aminophylline infusion.

Methotrexate can injure the central nervous system through several metabolic pathways; so different mechanisms of neurotoxicity have been proposed. The exact pathogenesis of IT-MTX neurotoxicity is not known. MTX promotes the release of adenosine from fibroblast and endothelial cells. Raised adenosine level causes dilation of cerebral vessels, alters the synaptic activity in CNS, and slows discharges of neurons which might account for some of its neurotoxicity. ${ }^{6}$ Aminophylline is a competitive antagonist of adenosine, replaces adenosine from receptors, and results in rapid and complete amelioration of neurological signs and symptoms. ${ }^{3,4}$ A few small case-series and case reports favour the use of aminophylline to treat this leukoencephalopathy with a high success rate. ${ }^{3,6}$ These results also echo their findings as $83.33 \%$ of patients showed rapid and complete recovery 48 hours after intravenous aminophylline infusion along with full neuroprotective strategies. After patients recovered from IT-MTX neurotoxicity, they were transferred back to the Oncology Department from PICU. Further management was decided by the oncology team.

This study has several inherent limitations like single-centre, small sample size, retrospective in nature. The strength is the largestcase series on the use of aminophylline in IT-MTXleukoencephalopathy. There is an urgent need for a randomised controlled trial on the treatment of this devastating complication of IT-MTX with a comprehensive neurologic assessment to verify the observation.

In summary, the intravenous aminophylline in children with intrathecal methotrexate-induced leukoencephalopathy serves as "salvageable-therapy" without causing any adverse effect of aminophylline.

\section{ETHICALAPPROVAL:}

The study protocol was approved by the Ethical Committee of The Indus Hospital (study IRB Number: IRD_IRB_2020_04_006 dated 13-04-2020).

\section{PATIENTS' CONSENT:}

This is a chart review of data that exists before submission of proposal to IRB. Therefore, patients' consents were not required but principles of responsible and ethical conduct of research werefollowed.

\section{CONFLICT OF INTEREST:}

The authors declared no conflict of interest.

\section{AUTHORS' CONTRIBUTION:}

WR: Data collection and analysis.

AH: Manuscript formatting and designing.

HS: Manuscript writing and formatting tables and figures.

$\mathrm{RU}$ : Literature search related to drugs.

$\mathrm{NJ}$ : Literature search related to neurotoxicity in oncology.

SM: Compiling and finalizing the manuscript.

\section{REFERENCES}

1. Bajwa MH, Tahir A, Manzoor I, Khan S, Bakkar MA, Mubashlr MW, et al. Epidemiological distribution of pediatric oncology in Lahore, Pakistan. Biomedica 2017; 33(4): 291-5.

2. Sandoval C, Kutscher M, Jayabose S, Tenner M. Neurotoxicity of intrathecal methotrexate: MR imaging findings. AJNR Am J Neuroradiol 2003; 24(9):1887-90.

3. Bernini JC, Fort DW, Griener JC, Kane BJ, Chappell WB, Kamen BA, et al. Aminophylline for methotrexate-induced neurotoxicity. Lancet 1995; 345(8949):544-7.

4. Inaba $\mathrm{H}$, Khan RB, Laningham $\mathrm{FH}$, Crews $\mathrm{KR}$, Pui $\mathrm{CH}$, Daw NC. Clinical and radiological characteristics of methotrexate-induced acute encephalopathy in pediatric patients with cancer. Ann Oncol Off J Eur Soc Med Oncol 2008; 
19(1):178-84.

5. Ganesan P, Bajpai P, Shah A, Saikrishnan P, Sagar TG. Methotrexate induced acute encephalopathy-occurrence on re-challenge and response to aminophylline. Indian J
Hematol Blood Transfus 2014; 30(Suppl 1):105-7.

6. Vezmar S, Becker A, Bode U, Jaehde U. Biochemical and clinical aspects of methotrexate neurotoxicity. Chemotherapy 2003; 49(1-2):92-104. 